\title{
Powder bed generation in integrated modelling of additive layer manufacturing of orthopaedic implants
}

\author{
Michal Krzyzanowski $^{1,2}$ • Dmytro Svyetlichnyy ${ }^{1}$ - Grace Stevenson ${ }^{3}$. \\ W. Mark Rainforth ${ }^{2}$
}

Received: 23 June 2015 / Accepted: 4 February 2016 / Published online: 24 February 2016

(C) The Author(s) 2016. This article is published with open access at Springerlink.com

\begin{abstract}
This paper presents an original model of powder bed generation developed within the frame of an integrated modelling approach for studying the interaction of physical mechanisms in additive layer manufacturing (ALM) of orthopaedic implants. The model is based on cellular automata (CA) approach and describes the relationship between moving particles of different sizes during deposition on a surface in three dimensions. The surface is defined by the horizontal two-dimensional CA on which particles fall and irreversibly stick to a growing deposit. The model allows for consideration of different restructuring cases when particles are allowed to rotate as often as necessary until achievement of a local minimum position. Changes in the packing density of the powder bed have been investigated numerically depending on technological parameters, such as particle size distribution, deposition rate and sequence of powder deposition. The model has been developed with the aim of merging to the finite element (FE)-based integrated model and is applicable to a different ranges of materials including metals and also non-metals.
\end{abstract}

Keywords Additive layer manufacturing · Powder bed formation $\cdot$ Cellular automata model $\cdot$ Integrated finite element-based modelling

Michal Krzyzanowski mkrzyzan@agh.edu.pl

1 Faculty of Metals Engineering and Industrial Computer Science, AGH University of Science and Technology, Mickiewicza 30, 30-059 Krakow, Poland

2 Department of Materials Science and Engineering, The University of Sheffield, Mappin Street, Sheffield S1 3JD, UK

3 JRI Orthopaedics Ltd, 18 Churchill Way, Chapeltown, Sheffield S35 2PY, UK

\section{Introduction}

Additive layer manufacturing (ALM) is an intensively developing field of research for the manufacture of bioresorbable scaffolds in tissue engineering for repair of skeletal defects [1]. Different additive manufacturing methods have been used to prepare scaffolds, including selective laser sintering (SLS), whereby an object is built layer by layer using powdered materials, radiant heaters with a computer-controlled laser [2], precision extrusion deposition (PED) and fused deposition moulding (FDM) [3, 4]. Conventional manufacturing techniques, such as compression moulding, have also been used to produce viable scaffolds for bone tissue engineering [5]. However, ALM methods enable effective control over the structure of the scaffold, which in turn dictates the geometry of the newly formed tissue. Moreover, the ability of SLS to manufacture anatomically shaped scaffolds with designed microstructure made of bioactive and bioresorbable composite materials at high filler loadings allows for fabrication of scaffolds with a high degree of geometric complexity and enables the direct conversion of the digital representation of any object into its physical realisation. The method also enables the development of patient and tissue-specific reconstruction strategies [6-9].

Over recent years, orthopaedic implant research has been driven by biological fixation, in which interfacial bonding between the implant and bone is generated by formation of a layer of biologically active material on the implant surface [10]. Implants made by ALM methods can have increased longevity by providing more secure fixation using tailored porosity, which cannot be achieved by conventional manufacturing techniques [11]. The presence of an optimised and selective porous structure can influence host bone regeneration by creating an environment which allows cell spreading, proliferation and subsequent bone formation, enabling 
integration of the implant into the body. It has been shown that bone ingrowth into implants with deliberately created and controlled porosity allows for the strength increase of such bone-implant composite by a factor of 3 to 6 [12].

SLS has the potential to construct bioactive implants due to its capability of processing polymers, ceramics, metals and their composites. Bioresorbable scaffolds with mechanical properties suitable for bone tissue engineering have been fabricated from polycaprolactone (PCL) and hydroxyapatite (HA) by SLS. HA is a calcium phosphate $(\mathrm{CaP})$ material that is chemically similar to the inorganic component of bone matrix and can promote apatite deposition and bone cell attachment [13]. It has been demonstrated that SLS can be used for fabrication of physically blended HA-poly (ether-ether-ketone) (PEEK) and HA-poly (vinyl alcohol) composites for tissue scaffold development with micropores on the scaffold surface [14]. The ultimate compressive strength and elastic modulus for HA-poly-(L-lactic acid) (PLLA) parts fabricated by SLS was found within the lower limits of reported values for cancellous bone [6]. The beneficial effect of HA in composite scaffolds significantly depends on their microstructure, achieved density in designed solid regions and porosity of the scaffolds, sometimes exhibiting conflicting results $[15,16]$. $\mathrm{CaPs}$ are brittle materials with poor mechanical properties in torsion and bending. There are limited published data on the compressive mechanical properties of PCL composites fabricated using SLS with different or near-full density in designed regions. However, it has been shown that loading ceramics up to $30 \%$ with polymer composites can improve their mechanical properties [17]. FE modelling of mechanical properties can be seen as an effective tool in computational design and direct digital manufacture of patient- and site-specific composite tissue-engineering constructs with tailored properties [18].

Faster and cheaper fabrication of permanent fixed Prosthodontics is expected with laser-assisted densification of multiple materials via ALM technology known as multimaterial laser densification (MMLD) [19, 20]. The process has been developed to fabricate artificial teeth made of multiple materials in one operation without part-specific tooling and human intervention. An understanding of thermal transient and residual stresses, and also the influence of changes in process parameters on generation of these stresses, is required for development of a reliable MMLD process for dental restorations. FE analysis has provided substantial insights into the development of the temperature gradients, as well as thermal resistant and residual stresses involved in the conversion of a powder bed to a dense body [21-25]. Warpage of the fabricated multimaterial components has also been studied using FE-based modelling. It has been shown that, among others, the effect of conversion of a powder compact to a dense solid is significantly important for achievement of reasonable prediction accuracy in the modelling results, but this was not considered in the majority of the models presented in the literature. For instance, the simulation that starts with powder elements predicts an asymmetric molten pool during laser heating and densification, while the simulation that starts with solid elements suggest a symmetric molten pool. The solid model also shows lower temperature gradients then those predicted using the powder model. The stress values derived from the solid model are lower than those from the powder one due to the smaller thermal gradients in the fabricated part [25]. Although both solid and powder models predict the same distortion profile, it has been shown that the degree of warpage is decreased when the material is changed from powder to solid during SLS process. This effect is due to the larger thermal conductivity of material in the solid state rather than in the powder one and, as a result, smaller temperature gradients in the part being fabricated. To address the mentioned deficiency in modelling of the powder bed transition to a dense solid, the present study develops an integrated FE-based modelling approach, applicable to a range of materials including metals and non-metals. The integrated model is comprised of submodels for specific mechanical, optical, thermal, metallurgical and chemical phenomena taking place during ALM of orthopaedic implants with controlled porosity and mechanical properties. Modelling of the powder bed formation using a cellular automata (CA) three-dimensional (3D) numerical approach is considered in the first part of the work.

\section{The role of powder bed}

Bioactive glass was developed in the late 1960s and the first glass composition shown to form biological fixation with bone was named $45 \mathrm{~S} 5$ or Bioglass ${ }^{\circledR}[26,27]$. This formulation has been used in a variety of commercial bone graft materials as it stimulates healing and induces regeneration of bone tissue [28]. Recent research has also demonstrated the potential of bioactive glass as a bone tissue scaffold material [27, 29]. Tissue scaffolds aim to support three-dimensional tissue formation by providing a structure for cell attachment and a microenvironment to promote regeneration. In the case of bioactive glass, the tissue scaffold is gradually dissolved and replaced by the regenerated bone, the formation of which is stimulated by resorption of the dissolution products from the glass. The following characteristics of a bioactive glass are important for the construction of bone tissue scaffolds, namely the rate of biodegradation, osteoconductive and bioactive abilities and an ability to deliver cells [30]. Bioactive glass material has also shown its osteoinductive abilities and can be formed into a highly porous structure, which is a valuable quality of a bone tissue scaffold material. It has also been reported that bioactive glass exhibits antibacterial effects due to the high aqueous $\mathrm{pH}$ created as dissolution progresses [31]. 
The metal and bioactive glass for production of orthopaedic implants and tissue scaffolds by ALM are supplied in powder form and subsequent densification, for example by sintering or melting, is a crucial step in these processes [32]. Particle sizes play an important role on sintering [27, 33, 34]. Smaller particles tend to sinter at a higher rate due to the increased surface area. For instance, it has been shown for Ti6Al4V powders that as particle size increases, the relevant sintering temperature increases accordingly [34]. Larger particle sizes induce a higher resistance to sintering. Moreover, it has been found recently that sintered density decreases with increasing particle size [35]; an effect that has been observed for a number of materials [33, 34]. Density is an important parameter of a bone tissue scaffold and a well-designed scaffold should have a density and compressive strength similar to that of bone [30]. In construction of bioactive glass bone tissue scaffolds, the direct impact of particle size on sintering behaviour is more complicated. It has been shown for $45 \mathrm{~S} 5$ Bioglass ${ }^{\circledR}$ powder that the sintering temperature decreased with increasing particle size from $32-38$ to $53-63 \mu \mathrm{m}$ range during heating at $5-10{ }^{\circ} \mathrm{C} / \mathrm{min}$ rate while increasing particle size from 53-63 to 90-106 $\mu \mathrm{m}$ range results in increasing of the sintering temperature. Figure 1 shows the observed influence of particle size on sintering temperature exhibiting Ushape curves. The phenomenon can be explained by some interaction effects between particle size and crystallisation during sintering $[36,53]$.

Laser-assisted ALM processes involve densification of materials and conversion of a powder bed to a dense body. A number of FE models have been generated in several previous studies of ALM technological processes; however, these simulations are based on either simplified models which assume that every element is initially solid before laser densification $[23,24]$ or on the models where the properties of the powder bed are simply treated as functions of temperature and the initial porosity (packing density) [25]. Due to a lack of relevant data, it is assumed in these calculations that a powder bed is composed of randomly packed, single-sized spheres [37]. It is known that the thermal properties of the powder bed are strong functions of the packing density of the deposited powder [37-39]. Moreover, not only single-sized particles but also a mixture of different powders with different particle sizes can be used for powder bed deposition depending on the particular ALM process conditions. The laser radiation due to diffraction effects penetrates deep into the powder bed. Normally, the distribution of penetrated intensity of laser radiation, $I$, is written as

$I=I_{L} \exp (-\gamma h)$,

where $\gamma$ is the extinction coefficient, $h$ is the coordinate directed into the powder bed and $I_{L}$ is the intensity of laser radiation at the surface of the powder bed [40]. The accuracy of

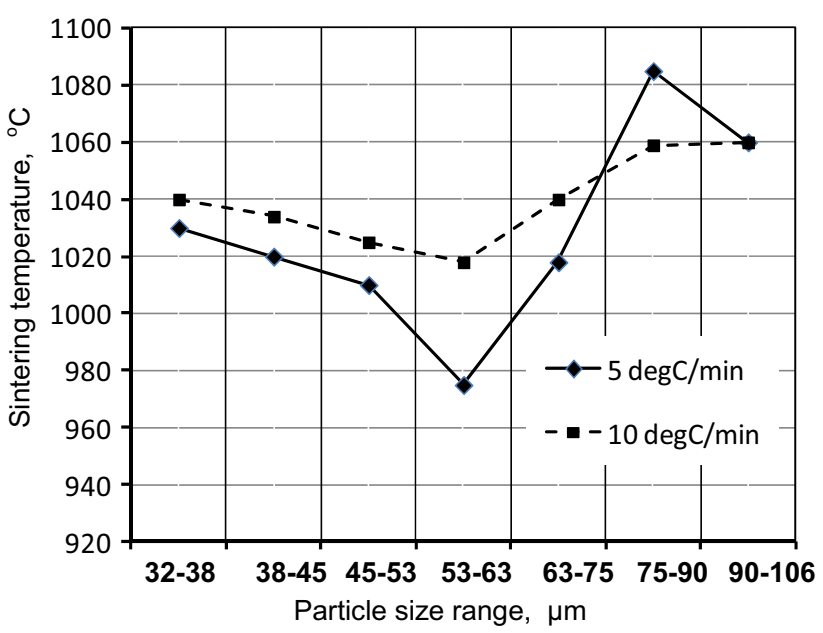

Fig. 1 Sintering temperature of $45 \mathrm{~S} 5$ Bioglass ${ }^{\circledR}$ powder for different particle size ranges and heating rates

predictions using Eq. (1) becomes significantly lower with decreasing particle size. Computer simulation of laser irradiation of powder beds is necessary to increase accuracy and optimise the laser-assisted ALM process. It has been shown using a 2D FE-based numerical solution of Helmholtz equation, taking into account the wave nature of light propagation, that the efficiency of penetration of laser radiation into a powder bed is strongly determined by the particle size, morphology and packed density [41]. The simulations were performed for different particle sizes and packed densities, which were incidentally placed in the computational area. In view of a large variety of powder dispersity and types of materials used, computer modelling of laser energy transport may give us an appropriate approach to predict the extinction coefficient, which is necessary for the modelling of heat and mass transport during ALM processes. However, modelling requires knowledge of particle size distribution, morphology and packed density which was considered but not predicted previously. To address this deficiency, the present study develops a model of powder bed formation to include the effects of restructuring during powder deposition in the ALM process. Furthermore, preliminary particle size distributions and the speed and sequence of powder delivery are considered in the present model. The developed model improves the accuracy of prediction with respect to powder bed packing density and morphology. In addition, this model can be embedded into an integrated FE model to simulate the penetration of laser radiation into, as well as thermal conductivity and thermal convection around a deposited powder bed.

\section{Model of powder bed generation}

The model of powder bed generation has been developed as part of an integrated multilevel modelling approach that is being developed to study the interaction of physical 
mechanisms in laser-assisted ALM of orthopaedic implants (Fig. 2). The integrated approach is considered to be useful for process development, especially when qualifying new materials and investigating different technological aspects.

The absorption properties of the powder bed and modelling of the heat source are considered within the optical submodel. They can be explained by the topology of powder beds and the oxidation of the particle surface layers [42]. The thermal submodel, dealing with modelling of temperature fields during propagation of the laser beam, considers the non-steady or steady state with convection formulations and requires determination of the boundary conditions, energy balance and heat transport mechanisms. The sintering/melting submodel deals with physical phenomena related to material transport. Sintering or melting consists of consolidation of a powder mixture at a temperature close to (in the case of sintering) or above the melting temperature (in the case of melting) of the material. During this stage, particulate material is converted into a dense body (densification stage). Wetting of solid phases by the liquid is extremely important, particularly when it comes to laser sintering of composites. Thus, the surface conditions and chemical reactions, such as oxidation, have to be considered in the chemical submodel. The approaches developed earlier for joint numerical solution of the heat and non-equilibrium mass transfer in gaseous and solid phases for investigation of alloying kinetics during laser treatment of metals offer possibilities to take into consideration elementary processes defining kinetics of gas-solid interactions such as molecule dissociation, chemisorptions of the dissociated atoms, energy transferred to the metal particles during the dissociation process and intensity of gas emission out of the metal $[43,44]$. A detailed finite element analysis using a physically based oxide-scale model can be a crucial aspect towards understanding and prediction of oxide-scale behaviour [45]. Metallurgical mechanisms, for example alloying when processing metal-metal powder mixtures or changes in the state of aggregation under semisolid conditions, are considered and modelled in the metallurgical submodel, taking into account the obtained history of the temperature field including heating and cooling rates.

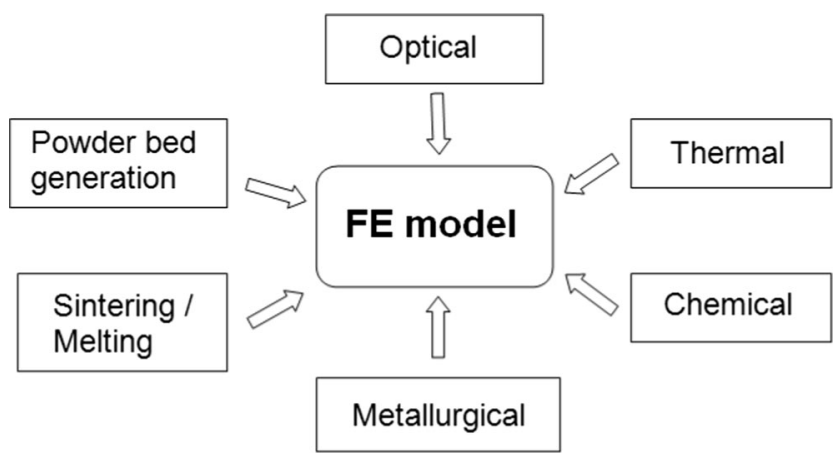

Fig. 2 Schematic representation of the integrated multilevel numerical approach applied for ALM process development

\subsection{Model assumptions and algorithm}

The model of powder bed generation described is based on the 2D CA modelling approach. Particles are considered as hard balls with diameters distributed within an arbitrary range of $R_{\min } \leq R \leq R_{\max }$. The deposit is grown on a steady basement, which is initially represented by a horizontal flat surface. The particles are added one at a time to the surface after determination of the particle's location. A new basement is formed by the initial surface plus distributed particles and can be considered as a horizontal projection of the $3 \mathrm{D}$ powder deposit. 2D CA elements are applied for the basement which contains quadratic cells. The sizes of the CA cells are chosen assuming that only one particle can be placed within a cell at the same level. Hence, the diagonal of a cell $c$ should be equal to the minimal particle diameter and the side of a cell. It is calculated in the following equation:

$c=\sqrt{2} R_{\min }$

In each CA cell, $n_{c}$ particles can be situated at different levels. The location of a cell in the CA space is described by two integer coordinates, $(i, j)$. Each CA cell contains information about the number of the particles inside the cell situated at different levels. The coordinates of the centre and the diameter are defined for each particle.

The algorithm of the powder bed generation model is presented in Fig. 3. The model considers an introduction of the particles either from a specified point or randomly across a specified area above the basement at a predefined rate. Then, the following three cases can be triggered, such as the case with no restructuring (called one contact model), the case when only partial, or a local, readjustment is allowed (called two contact model) and the case when multiple restructuring is allowed and the readjustment may eventually extend to a larger scale (called three-contact model). In the one-contact model, the particles stick at either their first contact with the deposit or with the basement. According to the second contact model, after contacting a particle in the deposit, the deposited particle moves downhill maintaining contact with the first contacting particle in the deposit until it contacts a second particle in the deposit or the basement. The particle is deposited onto the basement if no contact is made with the growing deposit. In the multiple restructuring model, the deposited particles move along the surface of the deposit, maintaining contact with it and reducing its potential energy. The movement is continued until the particle reaches on local minimum in contact with two of the particles on the deposit or reaches the basement. This model gives a close packed structure of the powder bed.

\subsection{One-contact model—no restructuring}

At each step, the vertical movement of a particle is considered. The one-contact model assumes that the falling particle is 


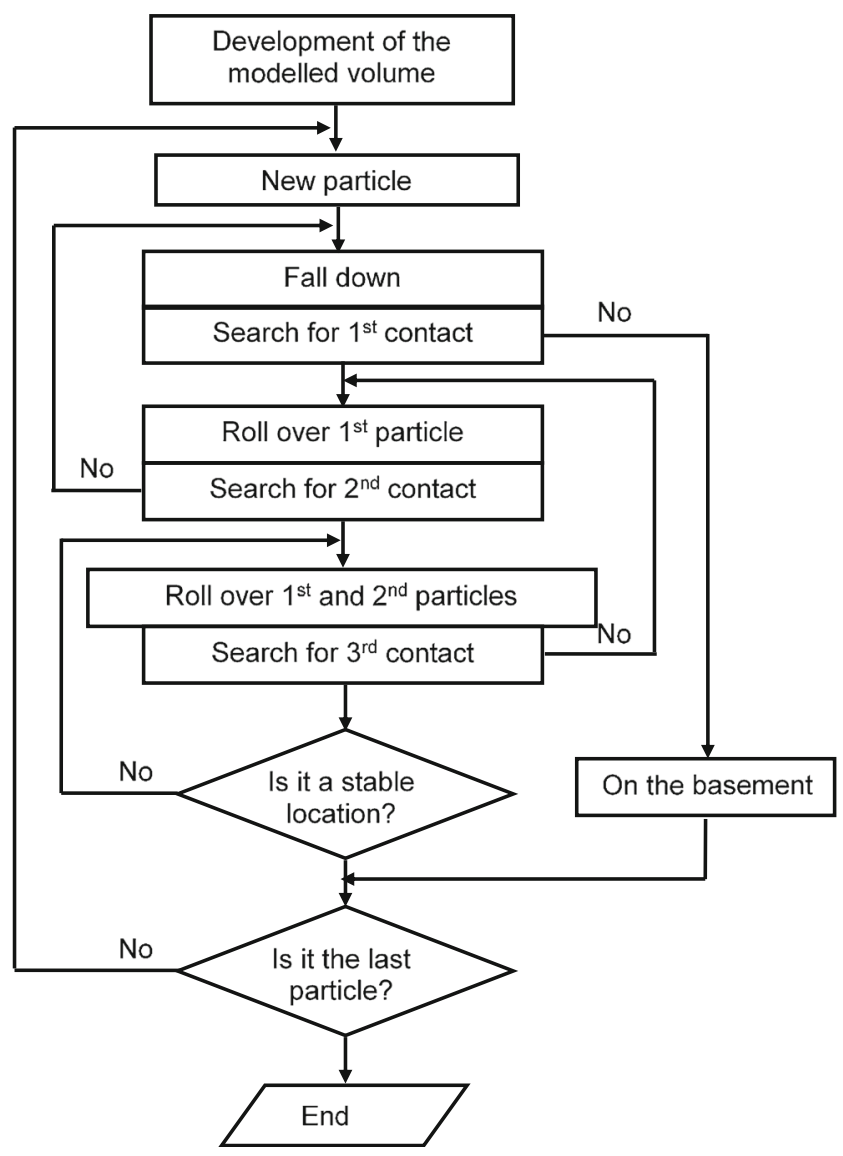

Fig. 3 Algorithm of the powder bed generation model

stopped either at the first contact with an already deposited particle or with the basement. If no contacting particle is found, the new particle is deposited on the basement surface. At each step, a random vertical trajectory and a particle size is selected by choosing the corresponding coordinates of the particle centre $\left(x_{c}, y_{c}\right)$ and the radius, $R_{c}$. The particle is considered to be added to the domain after determination of $z_{c}$. To detect the possible contact of the falling particle with previously deposited particles, only neighbouring cells are considered. The border coordinates of the region (neighbourhood) around a falling particle having coordinates, $\left(x_{c}, y_{c}\right)$, and radius, $R_{c}$, are calculated as $x_{c} \pm\left(R_{c}+R_{\max }\right)$ and $y_{c} \pm\left(R_{c}+R_{\max }\right)$, taking into consideration the domain boundaries: $x_{\min }, x_{\max }$, $y_{\min }, y_{\max }$ and the maximal particle radius, $R_{\max }$. The corresponding region of CA cells is defined in the following equations:

$i_{\min (\max )}=\operatorname{int}\left(\left(x_{c} \pm\left(R_{c}+R_{\max }\right)\right) / c\right)+1$

$j_{\min (\max )}=\operatorname{int}\left(\left(y_{c} \pm\left(R_{c}+R_{\max }\right)\right) / c\right)+1$

Deviation of the vertical trajectory of a falling particle from the position of an earlier deposited particle is analysed for each particle within the CA cells from the region. When the deviation is sufficiently small, the $z_{c}$ coordinate of the deposited particle is determined and the next cell is similarly analysed.
However, if the deviation exceeds the assumed critical value, the next particle within the same cell is analysed until the lowest particle is reached. The deviation, $l_{l}$, of the vertical trajectory of a falling particle is defined by the coordinates, $\left(x_{c}, y_{c}\right)$, of a previously deposited particle within the cell, $\left(x_{s}\right.$, , $\left.y_{s 1}\right)$, and is calculated by Eq. 4

$l_{1}=\sqrt{\left(x_{c}-x_{s 1}\right)^{2}+\left(y_{c}-y_{s 1}\right)^{2}}$

The falling particle is considered to have contact with a previously deposited particle within the cell when $l_{1}<R_{c}+$ $R_{s l}$, where $R_{c}$ and $R_{S I}$ are the radii of the falling and deposited particles, respectively. The difference between the levels of the two particles, $\Delta z$, is determined by Eq. 5

$\Delta z=\sqrt{\left(R_{c}+R_{s 1}\right)^{2}-l_{1}^{2}}$

The vertical coordinate, $z_{c}$, of the fallen particle is calculated by taking into consideration $\Delta \mathrm{z}$ and the current vertical coordinate of the centre of the falling particle, which is defined as $z_{c i}=z_{s l}+\Delta z$. The coordinates of the falling particle are compared to those of the previously deposited particle and if $z_{c i}>-$ $z_{c}$, then $z_{c}=z_{c i}$. It is initially assumed that $z_{c}=R_{c}$. The coordinate $z_{c}$ is calculated and the particle is considered to be deposited after the above analysis is completed for all cells from the defined neighbourhood. The procedure is then repeated for the next falling particle until a stop condition is reached.

\subsection{Two-contact model—partial restructuring}

In this model, the simulation proceeds as in the case of the one contact model in accordance with the algorithm described in Sect. 3.2 except that, after its first contact with an earlier deposited particle, the falling particle slides along the surface of the deposited particle until either it reaches a second deposited particle and stops its movement or slides down from the first particle. After determination of the first contact, the second contact is detected in the following way. Based on the coordinates of the falling and first contact particle, the vertical plane, in which the falling particle continues its movement, is defined. The centre of the falling particle moves in the defined plane along an arc, which is less than or equal to $90^{\circ}$, with the centre coincided with the centre of the first particle. The radius of the arc is equal to the sum of the corresponding particle radii, such as $R_{s I}+R_{c}$. The neighbourhood, where particles of the second contact can be located, is determined in a similar way to the one-contact model based on both ends of the arc. Each particle of every CA cell in this neighbourhood is tested for the second contact.

After the second contact, the distance between the falling and second particle will be defined as a sum of the corresponding radii $R_{s 2}+R_{c}$, where $R_{s 2}$ is the radius of the second 
Fig. 4 Deposition onto horizontal basement predicted using one-contact model for three consecutive stages. a-c Particles of the same size; $\mathbf{d}-\mathbf{f}$ particles of two different sizes
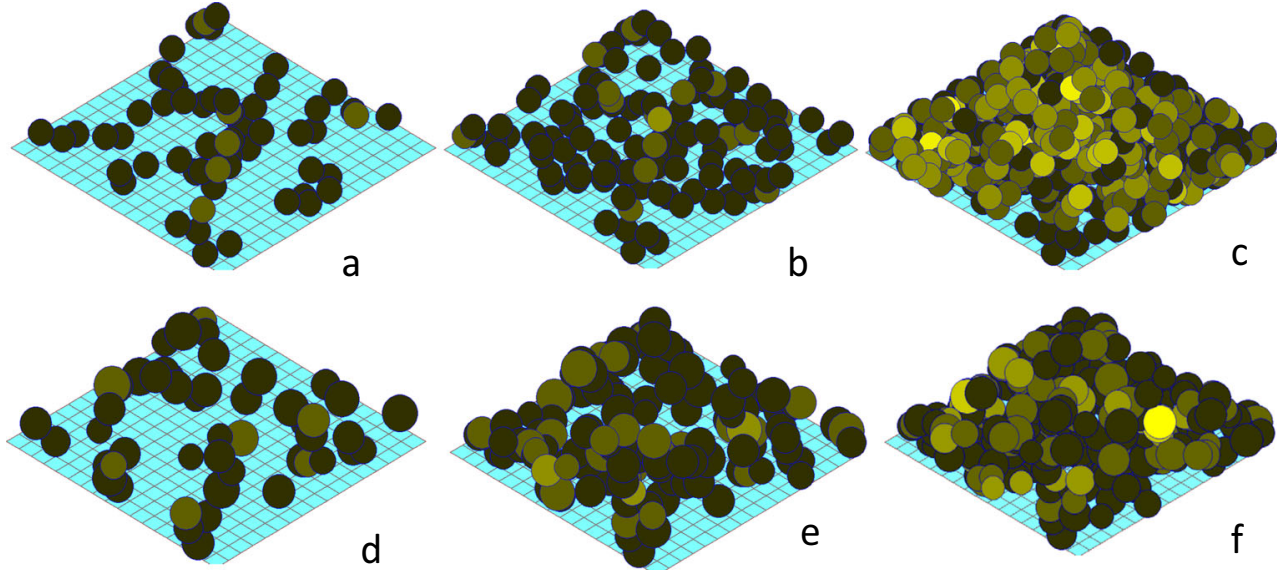

particle. The centre of the falling particle is laid on the sphere with the centre coincided with the centre of the second particle. The point of intersection of the abovementioned arc and the sphere is a possible centre of the falling particle after the second contact. The possible centre, nearest to the first contact, is considered as the second contact after testing all possible particles. The final position of the second contact is considered as the nearest to the first contact point of all possible centres. The falling particle slides from the first particle, if the final position is not determined. In such case, determination of the first contact is repeated with the initial coordinates at the end of the arc.

The intersection of the arc and the sphere is defined as the intersection of two circumferences laid in the vertical plane where the falling particle slides to, as defined earlier. The arc is part of the first circumference and the intersection of the sphere with the vertical plane is the second circumference. The radii and centre coordinates of these two circumferences are determined and the coordinates of intersections of the circumferences are calculated subject to the occurrence of such intersections. Thus, the centre of the falling particle will be located in these intersections situated on the arc, determining its possible final position in contact with two earlier deposited particles.

\subsection{Three-contact model—multiple restructuring}

A three-contact model allows for the most realistic representation of powder deposition in a strong gravity field. The model consists of the parts described for the one and twocontact models plus an additional part, where the third particle contact is calculated after establishing two previous contacts. The algorithm for this part of the model is similar to that for the determination of the second contact. The main difference is in the trajectory of the falling particle. In the two-contact model, the falling particle rolled over one particle and its movement was described by an arc of the circumference which was located in the vertical plane; however, in this part of the algorithm, the arc lays in the plane, which is defined by two particles. The normal to the plane is parallel to the line connecting the centres of these particles.

Firstly, the plane, the coordinates of the centre and the radius of the circumference are determined. The end of the arc is limited by the z-coordinate corresponding to the centre of the higher of the two mentioned particles. Contact with the highest particle should be lost beneath this point and the algorithm for the establishment of the second contact should be repeated again.

The neighbourhood, where particles of the third contact can be located, is similarly determined and each particle of every CA cell in this neighbourhood is tested for the third contact. The spheres with centre points that coincide with the centres of appropriate particles and the corresponding radii are determined, and the coordinates of intersections of the arc with the spheres are calculated as possible places of the third contact. The third contact is located at the point nearest to the location of the second contact after testing all possible
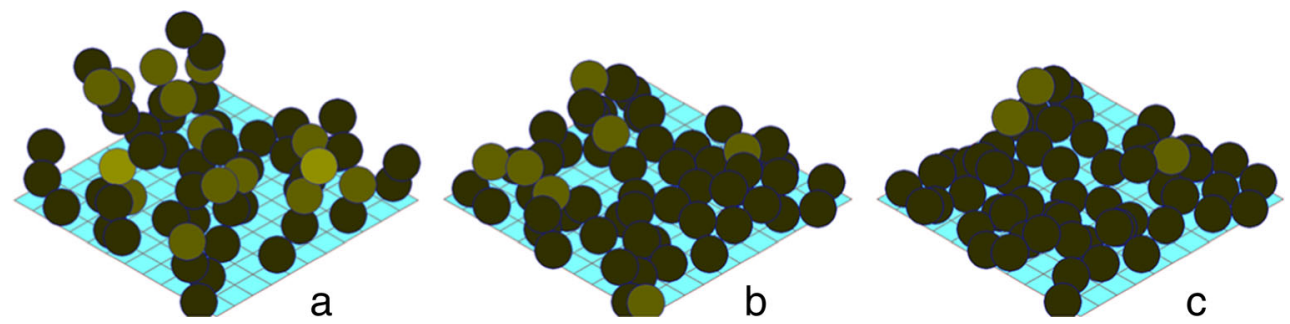

Fig. 5 Deposition of 60 particles onto horizontal basement predicted using different contact models. a one-contact model —no restructuring; $\mathbf{b}$ twocontact model — partial restructuring; $\mathbf{c}$ three-contact model — multiple restructuring (particle radius $15 \mu \mathrm{m}$ ) 


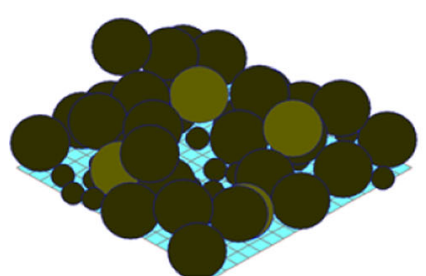

a

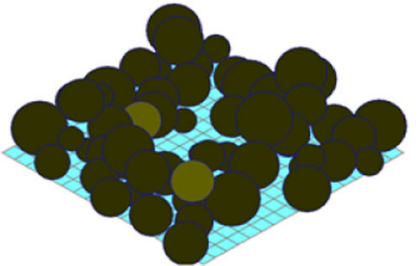

b

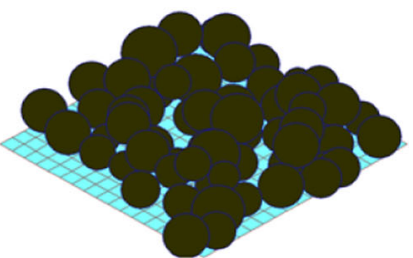

C
Fig. 6 Deposition of 60 particles onto a horizontal basement predicted using the three-contact (multiple restructuring) model for different particle size distributions. a Bimodal distribution with particle radii of
10 and $15 \mu \mathrm{m}$; b uniform distribution with particle radii of $10-25 \mu \mathrm{m}$; c Gaussian distribution with mean particle radius of $17.5 \mu \mathrm{m}$ and standard deviation of $2.5 \mu \mathrm{m}$ particles. If such a point is not defined, the particle slides further with subsequent determination of the second contact at the end of the arc.

\section{Results of numerical modelling}

The main result of the new CA-based model of powder bed generation is its capability to provide an effective numerical tool for generating desirable digital packing characteristics, such as various particle size distributions and packing densities, depending on the different deposition parameters described beneath. The obtained digital packing can be directly inputted into FE-based numerical models. It is particularly useful for an integrated multilevel modelling approach, which is being developed for studying the interaction of physical mechanisms in laser-assisted ALM of orthopaedic implants and predicting properties of materials that the model structure represents.

\subsection{General features}

To visualise the process of powder bed generation, typical examples of simulations carried out using the one-contact model for particles of the same and two different sizes are shown in Fig. 4.

An increase in deposit density can be clearly seen for the illustrated distributions of particles at three consecutive time points, (Fig. $4 \mathrm{a}-\mathrm{f}$ ). It can also be seen that the packing density is higher when smaller particles are deposited. Precise numbers for the packing densities will be given below; however, it should be mentioned that the observed disordered structure of the powder bed was stable in terms of the packing density for the given initial conditions. As the deposit grows, it may be that a particle does not exactly fit a vacancy within a CA cell due to the round-off errors of the numerical calculation and as such, even using a one-contact model with one particle size is enough to cause some irregularities in the packing. These irregularities are quickly amplified to reach the disordered dense structure. This is in agreement with real practice since one can never realise a process with rigorously mono-disperse particles. Thus, the simulated disordered structure is naturally obtained as long as one stays within the assumption of the model which neglects particle deformation. The increase of the deposit density from using different models with 1 to 3 contacts, as described in Sect. 3, can be clearly seen in Fig. 5, where simulation results are presented for the deposition of 60 particles. In the case of the models with restructuring, such as two or three contact models, the simulation proceeds after the first contact with a previously deposited particle. The falling particle slides along the surface of the contacted particle until it reaches either another deposited particle or the basement, in such way, increasing the packing density of the deposit.

\subsection{Different particle sizes}

The model with multiple restructuring (three-contact model) has been used to generate the random powder bed. Particles from a given size distribution (e.g. a bimodal, uniform or Gaussian distribution) are deposited one by one in randomly selected positions above the flat basement (Fig. 6).

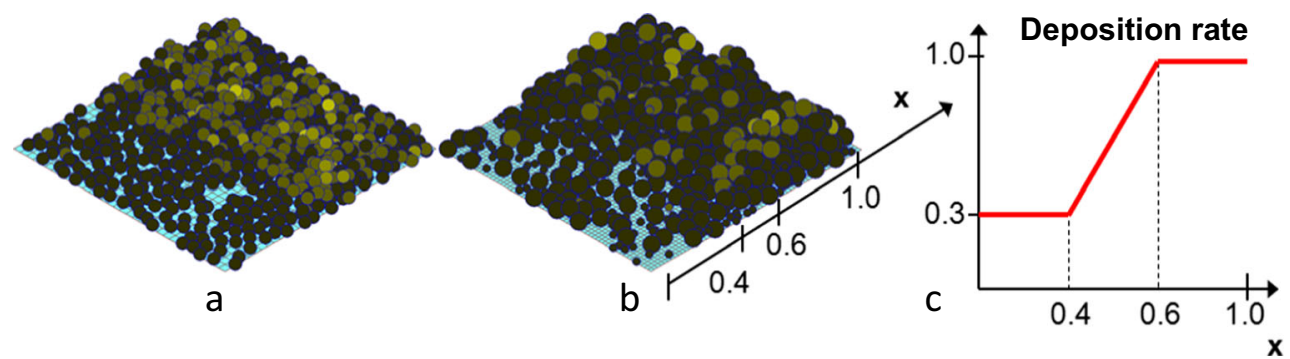

Fig. 7 Deposition of 1000 particles onto horizontal basement predicted using multiple restructuring model for the different powder delivering speed. a Particle size $-5 \mu \mathrm{m}$; b bimodal distribution, particle size -3 and $7.5 \mu \mathrm{m}$; $\mathbf{c}$ normalised delivery speed versus distance along the $x$-axis 
Fig. 8 Deposition of 300 larger particles (7.5- $\mu \mathrm{m}$ radius) on the top of smaller particles $(3-\mu \mathrm{m}$ radius) predicted using multiple restructuring model. a Number of $3-\mu \mathrm{m}$ particles - 700 ; b number of $3-\mu \mathrm{m}$ particles -1700
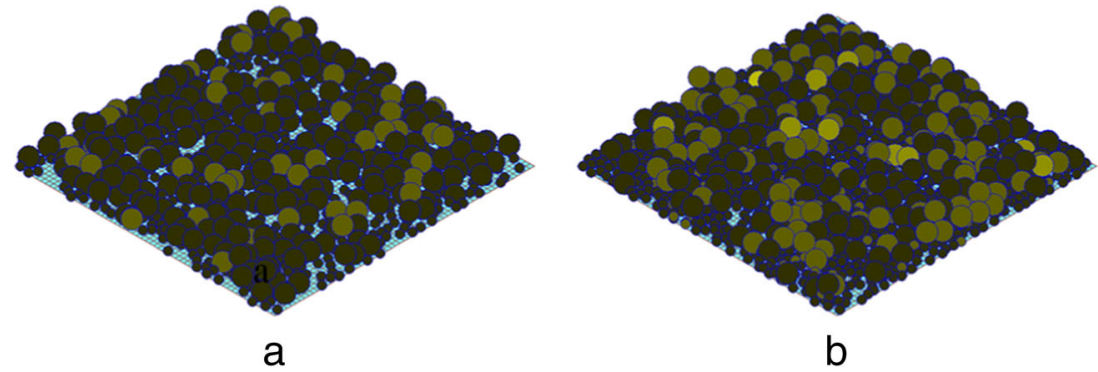

The newly introduced particle falls downward until it comes into contact with a stationary particle. Subsequently, the falling particle attempts to minimize its energy by rolling around the surface of the stationary particle and any other particle that it comes into contact with until it touches the third particle reaching the nearest local minimum. If no particle contact is found, the particle is deposited on the basement. The small number of deposited particles used in the simulation makes it easy to visualise differences in the structure of the generated deposits depending on the particle size distribution. Uniform and bimodal distributions were found to produce a less dense structure where characteristic "active zones" with higher porosity were formed by larger particles at the top of the deposited powder bed.

\subsection{Change of deposition rate and sequence of powder deposition}

It is also possible to control the structure of the powder bed by changing the deposition rate and sequence of particles being delivered. The generated structure is changed along $x$-axis during deposition of 1000 particles at different deposition rates, as shown in Fig. 7. Both the deposition rate and the distance along the $x$-axis have been normalised for convenience. The resultant structure of the deposit is illustrated for the case of equal size particles (Fig. 7a) and for the one of bimodal size distribution (Fig. 7b), while the change of deposition rate along $x$-axis is shown in Fig. 7c.

Changing the deposition sequence of powders, with different particle sizes, will inevitably have an impact on the structure of the powder bed. For illustration of the effect, the deposition of 300 particles having $7.5-\mu \mathrm{m}$ radius on top of smaller particles, with particle radius of $3 \mu \mathrm{m}$, has been predicted using the multiple restructuring model (Fig. 8).

To change the sequence of the deposition, different quantities of smaller particles (700 and 1700) have been deposited on top of 300 larger particles using the same model (Fig. 9). The possibility of taking into account the rate and sequence of powder deposition with different particle sizes during modelling of powder bed generation opens a new way for ALM process mapping. Such modelling offers opportunities to establish the relationship between process variables, such as material feed rate and beam powers, beam travel speeds and process outcomes such as microstructural features and melt pool dimensions. This approach can incorporate the physics of the ALM process, which deepens the understanding of the dependence of process outcomes on different variables.

\subsection{Deposit densities}

The density of the generated powder bed is calculated automatically after the simulation. The deposit density is obtained for a region within the generated powder bed well away from its boundaries, basement and upper surface formed by the uppermost layer of the deposited particles. The number of deposited particles is increased for this purpose to reduce the numerical errors. The packing density is calculated for the particles located within a rectangular cuboid positioned within the generated deposit and addressed as a representative cell further in the text (Fig. 10).

For this purpose, the top and bottom faces of the representative cell are located correspondingly at $0.75 h$ and $0.25 h$, where $h$ is the height of the generated deposit, while the side faces of the cell are situated accordingly to reduce an influence of the boundaries. The packing density is calculated as the

Fig. 9 Deposition of $\mathbf{a} 700$ and $\mathbf{b}$ 1700 smaller particles $(3-\mu \mathrm{m}$ radius) on top of 300 larger particles $(750-\mu \mathrm{m}$ radius) predicted using the multiple restructuring model

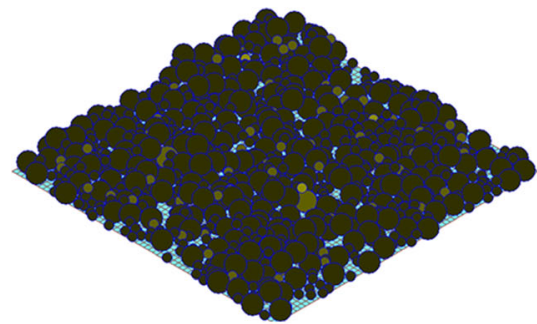

a

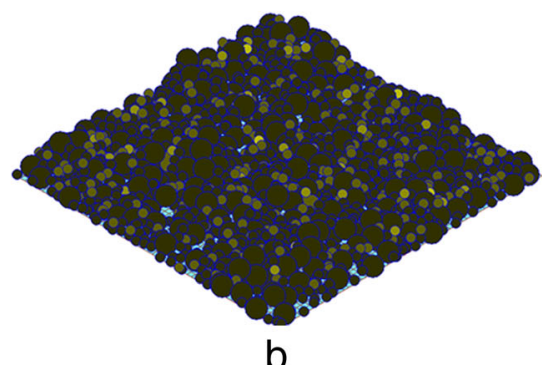


Fig. 10 Schematic illustration of the representative cell used for calculation of deposit density

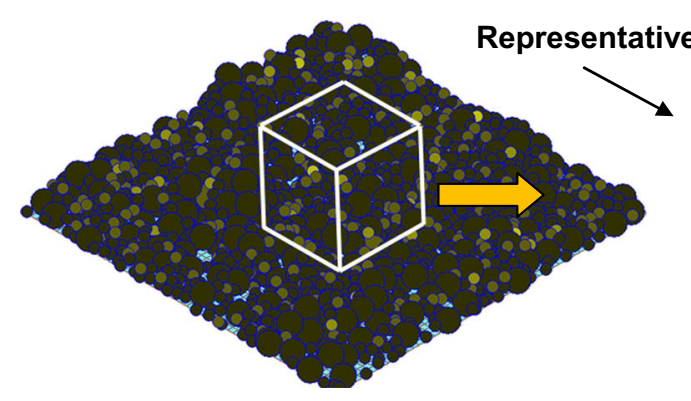

Representative cell
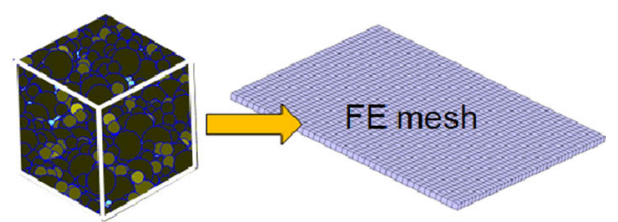

ratio of the total particle volume inside the representative cell to the volume of the cell. A particle with the centre located within the representative cell is considered to be within the cell. The deposit densities have been calculated on the basis of the deposits generated from 25,000-35,000 particles. For the case of the one-contact model with uniform 3- $\mu$ m particle radius, the calculated density was 0.1446 ; for two and threecontact models, the deposit density was defined as 0.4414 and 0.7325 , respectively. The deposits generated from the particles having uniform particle size distribution, with radiuses from 3 to $7 \mu \mathrm{m}$, exhibited higher calculated density values of 0.1464 , 0.4747 and 0.7927 for the one, two and three-contact models, respectively. The three-contact (multiple restructuring) model gives an almost maximally possible deposit density of 0.7405 , calculated for particles of a uniform size. Powder beds with higher densities can be obtained using particles of different sizes. The thermal properties of the powder bed will be estimated and treated in further consecutive stages of the integrated FE analysis on the basis of the packing density calculated at this stage.

\subsection{Verification of the powder bed generation model}

The model was verified by comparison of the simulation results with statistical results obtained using 3D X-ray computed tomography (XCT) scans allowing for quantification of the size, morphology, frequency and distribution of the particles in three-dimensional space. By using XCT systems with different resolutions, it has been possible to quantify the positions of the particle centres within the deposited powder bed and also to measure their true sizes and morphologies. The applied experimental technique has been described in details elsewhere [46]. Ti6A14V powder was chosen for the model verification because of the spherical shape of the particles (Fig. 11). The results of the particle quantification have shown that the average aspect ratio was 1.1 , which is very close to spherical.

After deposition, the coordinates of the centre and the corresponding diameters were obtained for all the deposited particles by using the XCT technique. Statistical results from $\mathrm{XCT}$ scans of the powder feedstock illustrate the measured size distribution of the particles (Fig. 12a) as well as the reconstructed image of the cylindrical experimental deposit
(Fig. 12b). All 14,523 identified particles were used for the modelling. The particles were mixed and randomly deposited across the specified cylindrical area of the same diameter (1.82 $\mathrm{mm})$. The multiple restructuring (three-contact) model was applied for the simulation and comparison (Fig. 12c). The deposit density can be defined as the ratio of the volume of all particles in the cylinder to the total cylinder volume. Thus, the height of the cylinder is inversely proportional to the deposit density and can be used for comparison. The height of the experimentally obtained deposit was $1.8093 \mathrm{~mm}$ while the height of the corresponding simulated deposit was $1.752 \mathrm{~mm}$. It can favour the conclusion that the average simulated deposit density is about $3.2 \%$ higher than the experimentally obtained one showing good agreement.

\section{Discussion and future work}

Application of the CA methodology for simulation of different materials phenomena and processes is increasing nowadays. The methodology is already used for modelling of solidification, dynamic and static recrystallisation [47], crack propagation, grain refinement and phase transformation [48, 49]. This new CA-based model of powder bed generation provides an effective method of generating desirable digital particle

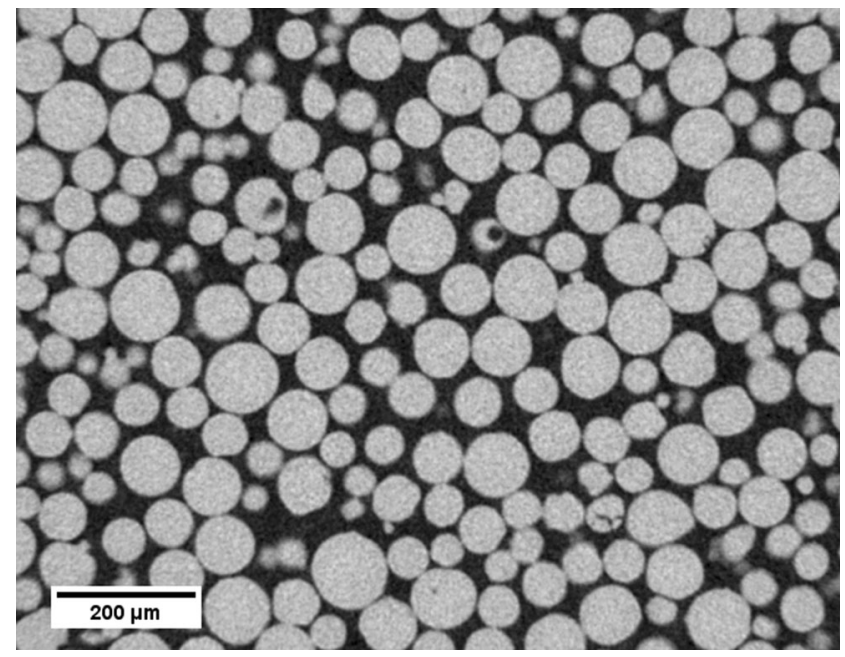

Fig. 11 The raw XCT image of Ti6Al4V particles illustrating their spherical shape 

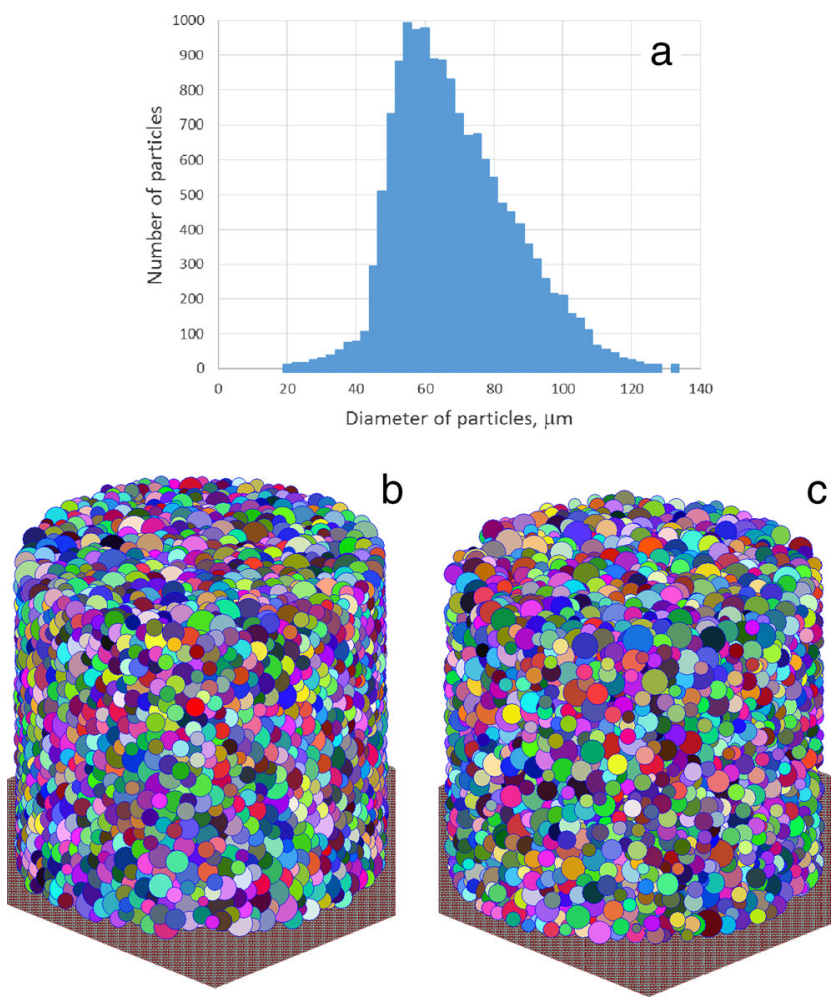

Fig. 12 Size distribution of the particles (a), XCT image showing example of the particle deposit (b) and an image of the corresponding powder deposit obtained using the multiple restructuring model (c)

packing for an integrated multilevel modelling approach being developed to study the interaction of physical mechanisms in laser-assisted ALM of orthopaedic implants. Using this method, a powder bed with desirable packing characteristics such as various particle size distributions and packing densities can be easily obtained. Particles can be introduced either from a specified point (point source), which is useful for estimation of friction coefficient, or randomly across a specified area (rain mode or hopper mode) above the basement at a predefined rate. The usefulness of the model is obvious for cases of laser-assisted ALM models, where knowledge of the packing structure and packing density is needed. The outcome of the model can be directly inputted into FE-based numerical models for studying and predicting properties of materials that the model structure represents. For example, calculation of the effective thermal conductivity and thermal radiation of the powder bed, and also thermal convection around the powder bed, is based on the relevant parameters of the packing density among others [23-25]. The description of laser radiation transport in dispersive powder beds deals with well-known difficulties. In many cases, they are not overcome by a simple estimation of the relevant packing density. In contrary to a solid material surface, the laser radiation penetrates deep into an area of porous powder beds due to diffraction effects. The accuracy of measurement and prediction of penetrated intensity of laser radiation is significantly lowered with decreasing metal particle size [40]. In view of the large variety of particle sizes and dispersivities of the applied powders, as well as the different materials used in laser-assisted ALM processes, computer simulation of laser irradiation in a powder bed, which explores the spatial distribution of radiation energy in a dispersive medium with particles, is necessary. The CAbased modelling approach described in this paper allows for prediction of such dispersive medium structure depending on the technological parameters of the ALM process.

Multimaterial laser-assisted ALM processes deal with different powder materials characterised by particle shape (morphology), size and distribution (granulometry). Particle shape can be classified as atomised (spherical or spheroidal), granular or flake. Most of the metal powders used in ALM can be considered as spherical for modelling purposes, and the structure of the powder bed for such materials can be well predicted
Fig. 13 a SEM image and b CA model representation of bioactive glass powder

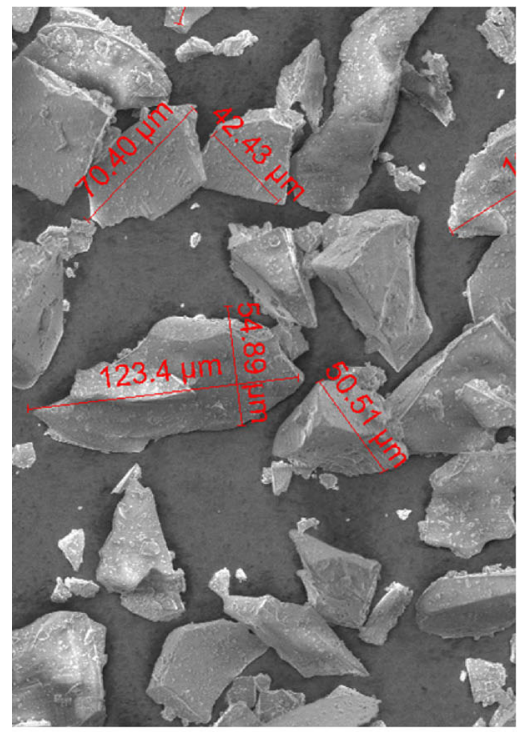

a

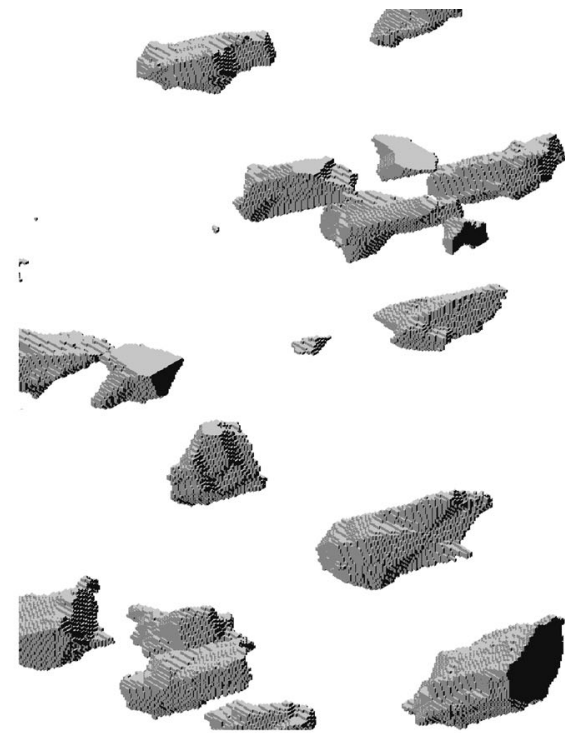

b 
using the described version of the CA model. However, not all materials used for ALM have spherical particle shapes, e.g. polymers. It has been shown that a typical bioactive glass powder is a granular material and the described CA modelling methodology opens up many new research opportunities, such as assumption of various shapes of both rigid and deformable particles (Fig. 13).

Validation of the applied models is a challenging task. So far, only a limited number of publications are relevant to validation of the packing algorithms [50]. X-ray tomography is a prospective tool to be used for validation of the packing structure of small particles generated by the different digital packing algorithms [51, 52].
Acknowledgments The support of the EPSRC under grant EP/ L505158/1, "Novel 3D Coating of Bioactive Glass and Metal Composites", and the National Science Centre Poland (grant no. DEC2013/09/B/ST8/00141) is greatly appreciated. The authors are particularly grateful for the input from JRI Orthopaedics Ltd and Glass Technology Services (GTS) Ltd. The authors would like to thank Dr. S. TammasWilliams for XCT and Dr Amit Rana for microscopic examinations, both from the University of Sheffield.

Open Access This article is distributed under the terms of the Creative Commons Attribution 4.0 International License (http:// creativecommons.org/licenses/by/4.0/), which permits unrestricted use, distribution, and reproduction in any medium, provided you give appropriate credit to the original author(s) and the source, provide a link to the Creative Commons license, and indicate if changes were made.

\section{References}

\section{Conclusions}

This research established that the developed CA-based model of powder bed generation is a useful numerical tool for generation of the desirable digital packing characteristics, such as various particle size distributions and packing densities, depending on different deposition parameters. These characteristics of the powder bed are important for its subsequent densification, for example by sintering or melting, which is a crucial step in production of orthopaedic implants and tissue scaffolds by additive layer manufacturing (ALM). The usefulness of the model is obvious for cases of laser-assisted integrated ALM models based on finite element (FM) methodology, where knowledge of the packing structure and packing density of the powder bed is required. The model considers an introduction of the particles either from a specified point or randomly across a specified area and can include cases with no, partial or multiple restructuring and calculates the movement of the particles until they reach the local minimum in contact with other particles of the deposit or the basement. Examples of the particles from a given size distribution, such as bimodal, uniform or Gaussian deposited one by one in randomly selected positions above the flat basement, were presented along with change of deposition rate and sequence of powder deposition. The model was verified showing good agreement with statistical results obtained using 3D X-ray computed tomography (XCT) allowing for quantification of the size, morphology, frequency and distribution of the particles in three-dimensional space. Most of the metal powders used in ALM can be considered as spherical for modelling purposes, and the structure of the powder bed for such materials can be well predicted using the described version of the CA model. However, the described CA modelling methodology opens up many new research opportunities, such as assumption of various shapes of both rigid and deformable particles, considering matrix alloys with different densities or mixed metal and non-metal powders.
1. Eshraghi S, Das S (2012) Micromechanical finite-element modelling and experimental characterisation of the compressive mechanical properties of polycaprolactone-hydroxyapatite composite scaffolds prepared by selective laser sintering for bone tissue engineering. Acta Biomater 8:3138-3143

2. Savalani MM, Hao L, Zhang Y, Tanner KE, Harris RA (2007) Fabrication of porous bioactive structures using the selective laser sintering technique. Proc Inst Mech Eng H 221:873-886

3. Domingos $\mathrm{M}$ et al (2011) Effect of process parameters on the morphological and mechanical properties of 3D bioextruded poly $(\varepsilon-$ caprolactone) scaffolds. Rapid Prototyp J 18(1):56-67

4. Lam CXF, Teoh SH, Hutmacher DW (2007) Comparison of the degradation of polycaprolactone and polycaprolactone- $(\beta-$ tricalcium phosphate) scaffolds in alkaline medium. Polym Int 56(6):718-728

5. Azevedo MC et al (2003) Development and properties of polycaprolactone/hydroxyapatite composite biomaterials. J Mater Sci Mater Med 14(2):103-107

6. Williams JM et al (2005) Bone tissue engineering using polycaprolactone scaffolds fabricated via selective laser sintering. Biomaterials 26(23):4817-4827

7. Melchels FPW, Domingos MAN, Klein TJ, Malda J, Bartolo PJ, Hutmacher DW (2011) Additive manufacturing of tissues and organs. Prog Polym Sci 37(8):1079-1104

8. Popov VK et al (2007) Laser technologies for fabricating individual implants and matrices for tissue engineering. J Opt Technol 74(9): 636-640

9. $\mathrm{Wu} \mathrm{G}$ et al (2008) Selective laser sintering technology for customised fabrication of facial prosthesis. J Prosthet Dent 100(1):56-60

10. Furlong RJ, Osborn JF (1991) Fixation of hip prostheses by hydroxyapatite ceramic coatings. J Bone Jt Surg Br 73(5):741-745

11. Mercuri LG, Wolford LM, Sanders B, White D, Hurder A, Herderson W (1995) Custom CAD/CAM total temporomandibular joint reconstruction system: preliminary multicenter report. J Oral Maxillofacial Surg 53:106-115

12. Hing KA, Best SM, Tanner KE, Bonfield W, Revell PA (2004) Mediation of bone ingrowth in porous hydroxyapatite bone graft substitutes. J Biomed Mater Res A 68:187-200

13. Hench LL, Polak JM (2002) Third-generation biomedical materials. Science 295(5557):1014-1017

14. Tan KH, Chua CK, Leong KF, Cheah CM, Cheang P, Abu Bakar MS, Cha SW (2003) Scaffold development using selective laser sintering of polyetheretherketone-hydroxyapatite biocomposite blends. Biomaterials 24(18):3115-3123 
15. Shor L et al (2007) Fabrication of three-dimensional polycaprolactone/hydroxyapatite tissue scaffolds and osteoblastscaffold interaction in vitro. Biomaterials 28(35):5291-5297

16. Guarino $\mathrm{V}$ et al (2008) The role of hydroxyapatite as solid signal on performance of PCL porous scaffolds for bone tissue regeneration. J Biomed Mater Res B: Appl Biomater 86B(2):548-557

17. Eosoly $\mathrm{S}$ et al (2010) Selective laser sintering of hydroxyapatite/ poly- $\varepsilon$-caprolactone scaffolds. Acta Biomater 6(7):2511-2517

18. Eshraghi S, Das S (2010) Mechanical and microstructural properties of polycaprolactone scaffolds with one-dimensional, two-dimensional, and three-dimensional orthogonally oriented porous architectures produced by selective laser sintering. Acta Biomater 6(7):2467-2476

19. Dai K, Shaw L (2002) Distortion minimization of laser-processed components through control of laser scanning patterns. Rapid Protot J 8(5):270-276

20. Li XX, Wang JW, Augustine A, Shaw LL, Marcus HL, Cameron TB (2001) Microstructure evaluation for multi-materials laser densification of dental porcelains. In: Bourell DL, Beaman JJ, Crawford RH, Marcus HL, Barlow JW (eds) Proceedings of the 12th Annual SFF Symposium. The University of Texas, Austin, pp 195-202

21. Matsumoto M, Shiomi M, Osakada K, Abe F (2002) Finite element analysis of single layer forming on metallic powder bed in rapid prototyping by selective laser processing. Int J Mach Tools Manuf 42:61-67

22. Dong L, Makradi A, Ahzi S, Remond Y (2007) Finite element analysis of temperature and density distributions in selective laser sintering process. Mater Sci Forum 553:75-80

23. Dai K, Shaw L (2003) The size effect on stresses and distortion of laser processed multi-material components. Metall Mater Trans A 34A:1133-1145

24. Dai K, Shaw L (2001) Thermal and stress modelling of multimaterial laser processing. Acta Mater 49:4171-4181

25. Dai K, Shaw L (2004) Thermal and mechanical finite element modeling of laser forming from metal and ceramic powders. Acta Mater 52:69-80

26. Hench LL (2006) The story of Bioglass®. J Mater Sci Mater Med 17:967-978

27. Jones JR, Clare AG (2012) Bio-glasses: an introduction. Wiley, New York

28. Hoppe A, Guldal N, Boccaccini AR (2011) A review of the biological response to ionic dissolution products from bioactive glasses and glass-ceramics. Biomaterials 32:2757-2774

29. Rahaman MN, Day DE, Bal BS, Fu Q, Jung SB, Bonewald LF, Tomsia AP (2011) Bioactive glass in tissue engineering. Acta Biomater 7(6):2355-2373

30. Chen OZ, Thompson ID, Boccaccini AR (2006) 45S5 Bioglass ${ }^{\circledR}-$ derived glass-ceramic scaffolds for bone tissue engineering. Biomaterials 27(11):2414-2425

31. Hu S, Chang J, Liu M, Ning C (2009) Study on antibacterial effects of 45S5 Bioglass ®. J Mater Sci Mater Med 20:281-286

32. Deb S, Mandegaran R, Di Silvio L (2010) A porous scaffold for bone tissue engineering/45S5 Bioglass ${ }^{\circledR}$ derived porous scaffolds for co-culturing osteoblasts and endothelial cells. J Mater Sci Mater Med 21(3):893-905

33. Chang HN, Pan J (2007) Sintering of particles of different sizes. Acta Mater 55:813-824

34. Robertson IM, Schaffer GB (2009) Some effects of particle size on the sintering of titanium and a master sintering curve model. Metall Mater Trans A 40(8):1968-1979
35. Francis JSC, Cologna M, Raj R (2012) Particle size effects in flash sintering. J Eur Cer Soc 32(12):3129-3136

36. Lefebvre L, Gremillard L, Chevalier J, Zenati R, BernacheAssolant D (2008) Sintering behaviour of 45S5 bioactive glass. Acta Biomater 4(6): 1894-1903

37. Sih SS, Barlow JW (1995) Emissivity of powder beds. In: Marcus H, Beaman J, Bourell D, Barlow J, Crawford R (eds) Proceedings of the 6th Annual SFF Symposium. The University of Texas, Austin, pp 402-408

38. Poirier DR, Geiger GH (1994) Transport phenomena in materials processing. The Minerals, Metals and Materials Society, Warrendale

39. Sih SS, Barlow JW (1995) The prediction of the thermal conductivity of powders. In: Marcus H, Beaman J, Bourell D, Barlow J, Crawford R (eds) Proceedings of the 6th Annual SFF Symposium. The University of Texas, Austin, pp 397-401

40. Kostenkov SN, Kharanzhevskiy EV, Krivilev MD (2012) Determination of characteristics of laser radiation interaction with nanocomposite powder materials. Phys Met Metallogr 113:93-97

41. Kharanzhevskiy E, Kostenkov S (2014) Modelling of laser radiation transport in powder beds with high-dispersive metal particles. J Alloys Compd 586:5246-5249

42. Luo J, Pan H, Kinzel EC (2014) Additive manufacturing of glass. J Manuf Sci Eng 136(6):061024. doi:10.1115/1.4028531, 6

43. Kryzhanovski M (1996) Validation of thermal-kinetic-diffusion model for arc plasma surface hardening of steel. Steel Res 67(6): 247-252

44. Pietrzyk M, Kryzhanovski M, Okara S, Parchomenko V (1996) Thermal-diffusion finite-element analysis of nitriding process for arc plasma surface hardening of steels. J Mater Process Technol $56: 412-421$

45. Krzyzanowski M, Beynon JH, Farrugia DCJ (2010) Oxide scale behavior in high temperature metal processing. Wiley-VCH Verlag GmbH \& Co. KGaA, Germany

46. Tammas-Williams S, Zhao H, Léonard F, Derguti F, Todd I, Prangnell PB (2015) XCT analysis of the influence of melt strategies on defect population in Ti-6Al-4V components manufactured by selective electron beam melting. Mater Charact 102:47-61

47. Svyetlichnyy DS (2012) Simulation of microstructure evolution during shape rolling with the use of frontal cellular automata. ISIJ Int 52:559-568

48. Svyetlichnyy DS (2013) Simulation of grain refinement by cellular automata. Comp Mater Sci 77:408-416

49. Svyetlichnyy DS, Mikhalyov AI (2014) Three-dimensional frontal cellular automata model of microstructure evolution - phase transformation module. ISIJ Int 54:1386-1395

50. Jia X, Gan M, Williams RA, Rhodes D (2007) Validation of a digital packing algorithm in predicting powder packing densities. Powder Technol 174:10-13

51. Lin CL, Miller JD (2000) Network analysis of filter cake pore structure by high resolution X-ray microtomography. Chem Eng J 77:79-86

52. X. Jia, N. Gopinathan, R.A. Williams, C.N. Eberhardt and A.R. Clarke, X-ray microtomography facilitated modelling of microstructures, In: Proc. 2nd World Congr. on Industrial Process Tomography, Hannover, Germany, 2001.

53. A. Hepp, The effect of bioglass particle size on sintering. Thesis completed in partial fulfilment of the requirements for the Alfred University Honors Program, Alfred University, NY 14802, USA, URI: http://hdl.handle.net/10829/4407. 